\title{
Hybrid Pressure Retarded Osmosis-Membrane Distillation System for Power Generation from Low-Grade Heat: Thermodynamic Analysis and Energy Efficiency
}

\author{
Shihong Lin, ${ }^{\dagger}$ Ngai Yin Yip, ${ }^{\dagger}$ Tzahi Y. Cath, ${ }^{\ddagger}$ Chinedum O. Osuji, ${ }^{\dagger}$ and Menachem Elimelech ${ }^{*}{ }^{\dagger}$ \\ ${ }^{\dagger}$ Department of Chemical and Environmental Engineering, Yale University, New Haven, Connecticut 06520-8286, United States \\ ${ }^{\ddagger}$ Department of Civil and Environmental Engineering, Colorado School of Mines, Golden, Colorado 80401-1887, United States
}

Supporting Information

\begin{abstract}
We present a novel hybrid membrane system that operates as a heat engine capable of utilizing low-grade thermal energy, which is not readily recoverable with existing technologies. The closed-loop system combines membrane distillation (MD), which generates concentrated and pure water streams by thermal separation, and pressure retarded osmosis (PRO), which converts the energy of mixing to electricity by a hydro-turbine. The PRO-MD system was modeled by coupling the mass and energy flows between the thermal separation (MD) and power generation (PRO) stages for heat source temperatures ranging from 40 to $80^{\circ} \mathrm{C}$ and working concentrations of 1.0, 2.0, and $4.0 \mathrm{~mol} / \mathrm{kg} \mathrm{NaCl}$. The factors controlling the energy efficiency of the heat engine were evaluated for both limited and unlimited mass and heat transfer kinetics in the thermal separation stage. In both cases, the relative flow rate between the MD permeate (distillate) and feed streams is identified as an important operation parameter. There is an optimal relative flow rate that maximizes the overall energy efficiency of the PRO$\mathrm{MD}$ system for given working temperatures and concentration. In the case of unlimited mass and heat transfer kinetics, the energy efficiency of the system can be analytically determined based on thermodynamics. Our assessment indicates that the hybrid PRO-MD system can theoretically achieve an energy efficiency of $9.8 \%$ ( $81.6 \%$ of the Carnot efficiency) with hot and cold working temperatures of 60 and $20^{\circ} \mathrm{C}$, respectively, and a working solution of $1.0 \mathrm{M} \mathrm{NaCl}$. When mass and heat transfer kinetics are limited, conditions that more closely represent actual operations, the practical energy efficiency will be lower than the theoretically achievable efficiency. In such practical operations, utilizing a higher working concentration will yield greater energy efficiency. Overall, our study demonstrates the theoretical viability of the PRO-MD system and identifies the key factors for performance optimization.
\end{abstract}

\section{INTRODUCTION}

The advancement of clean and renewable alternative power sources is necessary for the global shift to a sustainable energy future. ${ }^{1}$ Industrial waste heat and geothermal energy represent abundant and widely available energy sources that are currently underutilized. The industrial sector discharges approximately one-third of the energy consumed as thermal losses directly to the atmosphere or to cooling systems. ${ }^{2}$ Worldwide, the rejected heat amounts to $\sim 9,400 \mathrm{TWh} / \mathrm{y}$, based on the annual global industrial energy consumption of $\sim 28,000$ TWh. ${ }^{3}$ However, this energy is of varying quality, with most of the waste heat in streams below $150{ }^{\circ} \mathrm{C}$ or dissipated as radiation. ${ }^{4}$

While waste heat above $150{ }^{\circ} \mathrm{C}$ can be readily utilized to produce electricity using the conventional steam Rankine cycle and mid-temperature streams between 80 and $150{ }^{\circ} \mathrm{C}$ can be captured with binary cycle power plants, ${ }^{5-8}$ there are presently no established technologies to convert low-temperature $(<80$ ${ }^{\circ} \mathrm{C}$ ) waste heat into useful work. In the U.S., the total waste heat is $\sim 3,100 \mathrm{TWh} / \mathrm{y},{ }^{9}$ but only an estimated $6-10 \%(\sim 180-310$ $\mathrm{TWh} / \mathrm{y}$ ) is of adequately high quality for power generation with existing technologies. ${ }^{4,10}$ Therefore, low-grade industrial waste heat still represents a sizable energy source that can potentially be harnessed for electricity generation.

A vast amount of thermal energy, $\sim 1.5 \times 10^{12} \mathrm{TWh}^{11}$ is housed in the Earth's crust. The quality of geothermal energy, on average, increases with depth at $30{ }^{\circ} \mathrm{C} / \mathrm{km}$, with the temperature gradient heavily dependent on the geographical location and geological formation. ${ }^{7}$ Geothermal power plant developments are typically confined to locations with favorable shallow well sites, as the high temperatures needed for power generation with existing methods would otherwise necessitate the drilling of deep wells that are cost-prohibitive. ${ }^{7,12}$ Even though the geothermal energy reservoir is enormous, the worldwide geothermal power generation potential is assessed to be only $\sim 570-1200 \mathrm{TWh} / \mathrm{y} .{ }^{12,13}$ The development of technologies that can efficiently convert low-temperature heat streams into electricity will, therefore, allow us to gain greater

Received: November 20, 2013

Revised: April 7, 2014

Accepted: April 11, 2014

Published: April 11, 2014 
and wider access to the huge potential of geothermal energy, as well as to tap into a larger share of industrial waste heat.

The osmotic heat engine (OHE) is a promising technology that can produce electricity from low-grade heat sources. ${ }^{14}$ The closed-looped process comprises a power generation stage that converts the energy released from the controlled mixing of two solutions of different concentrations into useful work and a thermal separation stage where low-temperature heat energy is used to regenerate the low and high concentration solutions, which are then recirculated back to the energy production stage. ${ }^{15}$ Pressure retarded osmosis (PRO) and reverse electrodialysis (RED) are two membrane-based technologies that can be employed in the energy production stage. ${ }^{14,16}$ PRO utilizes the osmotic flow of water through semipermeable membranes from a low concentration solution into a high concentration solution to drive a hydro-turbine, thereby generating electricity. ${ }^{17-19}$ RED uses an alternating series of ion-selective membranes that allow only ionic species to diffuse down their concentration gradients. Electricity is produced when the ionic flow is converted into electrical current by redox reactions that occur at the end electrodes. ${ }^{20-22}$

The high concentration solution can be composed of thermolytic salts such as ammonia-carbon dioxide, ${ }^{15,23}$ trimethylamine, ${ }^{24}$ or switchable polarity solvents ${ }^{25,26}$ that can be thermally separated from the mixed solution using low grade heat. Alternatively, nonthermolytic salts, for example, sodium chloride and magnesium sulfate, can be employed to prepare the high concentration solutions. ${ }^{27,28}$ For solutions with nonvolatile solutes, membrane distillation (MD), an emerging thermal separation process using hydrophobic microporous membranes, can employ low-temperature heat in the thermal separation stage to regenerate the low and high concentration solutions. ${ }^{29-31}$

The OHE has the potential to unlock a substantial quantity of low-grade heat from industrial and geothermal sources and contribute to diversifying the global energy portfolio. The effectiveness of the processes that make up the technology has been individually studied to some extent. For example, recent publications have looked at the thermodynamic efficiency and practical potential of RED, ${ }^{32,33}$ the energy efficiency of PRO, ${ }^{18}$ and the thermodynamic limits of module-scale MD. ${ }^{34}$ To achieve a more holistic assessment of the viability of OHE to convert low-grade heat to usable energy and enable comparisons with competing technologies (e.g., thermoelectric heat engines or binary cycles engines), system-level analyses on OHE are required.

In this study, we conduct a systematic analysis of an OHE employing PRO for power generation and MD for working solutions regeneration. The analysis was performed on a module-scale system to assess the potential of the process by examining the thermodynamic limit of the energy conversion efficiency and to identify the major factors that control system performance. The analysis was carried out, first, by examining the performance and energy efficiency of the thermal separation stage (MD), which regenerates the low and high concentration streams using low-grade heat, and then by investigating the power output of the energy production stage (PRO), which converts the energy of mixing to useful work. The energy efficiency of the OHE was evaluated under several mass and heat transfer operating regimes in the thermal separation stage. The theoretically achievable energy efficiency of the OHE is compared to the Carnot efficiency, and the viability of the technology to harvest low-grade heat is discussed.

\section{MODEL DEVELOPMENT}

System Overview. A schematic diagram of the hybrid OHE system using $\mathrm{MD}$ for thermal separation and PRO for power generation is shown in Figure 1. In the power generation stage,

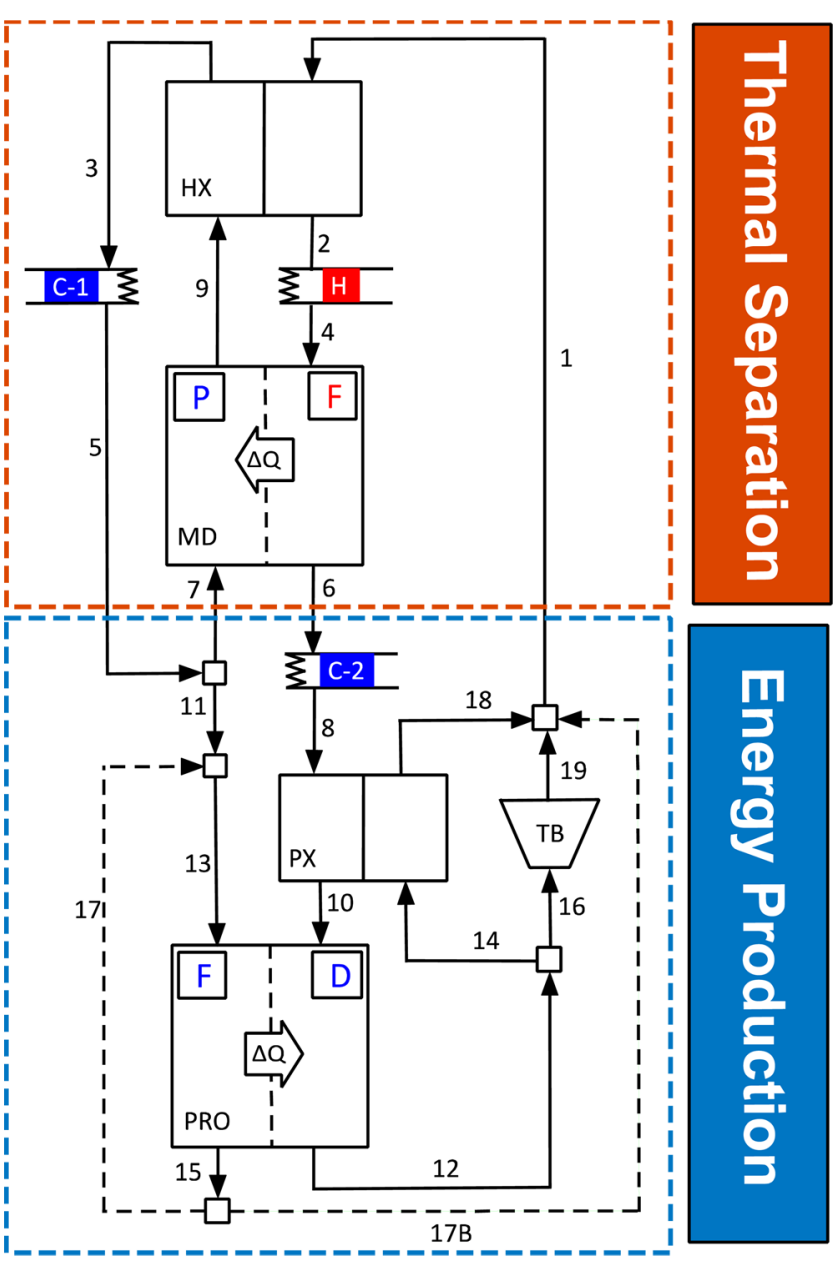

Figure 1. Schematic diagram of a PRO-MD hybrid system for harvesting low-grade heat energy. The system consists of (i) a thermal separation component that includes a membrane distillation (MD) module and a heat exchanger (HX) and (ii) a power generation component that includes a pressure retarded osmosis (PRO) module, a pressure exchange (PX), and a turbine (TB). The numbers represent the streams that are defined by the mass flow rate, the temperature, and the solute concentration. The " $\mathrm{H}$ " (in red) stands for an ideal constanttemperature heat source, whereas the "C1" and "C2" (in blue) represent ideal constant-temperature heat sinks. The " $\mathrm{P}$ " and " $\mathrm{F}$ " in the MD module symbolize the permeate (distillate) and feed channels, respectively. The "F" and "D" in the PRO module stand for the feed solution and draw solution channels, respectively. Stream 17B is optional as explained in Supporting Information.

controlled mixing of the diluted and concentrated streams via osmosis occurs across the PRO membrane to increase the flow rate of the pressurized concentrated stream. Useful energy is generated by the hydro-turbine (TB) by depressurizing part of the mixed solution at high pressure, while a pressure exchanger (PX) is employed to maintain a steady state operation. The depressurized mixed solution is then separated with MD using thermal energy to regenerate the diluted and concentrated streams. A heat exchanger ( $\mathrm{HX})$ is utilized in the thermal separation stage to recover the latent heat accumulated in the 
effluent of the MD permeate (distillate) stream to enhance the energy efficiency of thermal separation. The different streams in Figure 1 and their properties are listed in Table S1 of the Supporting Information.

Overall, the hybrid system absorbs heat from the heat source (red " $\mathrm{H}$ " in Figure 1), converts part of the absorbed heat to useful work through the turbine, and releases the remaining heat to the heat sinks (blue "C-1" and "C-2" in Figure 1), which reflects the working principle of a generic heat engine. We will explicate the working mechanism of the hybrid PRO-MD system by first explaining the thermal separation stage, which includes the MD and the $\mathrm{HX}$, and then elucidating the power generation stage, which includes the PRO, the PX, and the turbine.

For convenience of discussion, hereafter we denote stream $i$ as $S_{i}$, with mass flow rate, temperature, and salt concentration of $Q_{i}$, $T_{i}$, and $C_{i}$, respectively. The main stream of the hybrid system is $S_{1}$, which is the depressurized mixed solution from the TB and the PX, and is one of the three streams $\left(S_{1}, S_{8}\right.$, and $\left.S_{11}\right)$ that connect the thermal separation and power generation stages. The flow rate of $S_{1}$ represents the overall scale of the system. In addition, we define $C_{1}$ as the working concentration of the hybrid system.

Thermal Separation Stage. $S_{4}$ at the temperature of the heat source $\left(T_{\mathrm{H}}\right)$ and $S_{7}$ at the temperature of the heat sink $\left(T_{\mathrm{C}}\right)$ enter the MD module as the feed and permeate solutions, respectively. The temperature gradient across the hydrophobic membrane induces a partial vapor pressure difference that drives the water vapor from the feed solution to the permeate solution. For the entire module, the trans-membrane (vapor) flow rate $\Delta Q_{\mathrm{MD}}$ is equal to the reduction of feed flow rate (from $Q_{4}$ to $Q_{6}$ ) and the increase of the permeate flow rate (from $Q_{7}$ to $Q_{9}$ ) due to mass balance. For the permeate loop (including $S_{3}, S_{5}, S_{7}$, and $S_{9}$ ), the mass input is the trans-membrane vapor flux and the mass output is $S_{11}$. Therefore, steady state operation necessitates that $\Delta Q_{M D}$ equals $S_{11}$. We define the mass recovery rate, $\gamma$, as the ratio between the trans-membrane flow rate and the influent feed flow rate:

$$
\gamma \equiv \frac{\Delta Q_{\mathrm{MD}}}{Q_{4}}=\frac{Q_{11}}{Q_{4}}
$$

Heat is transferred across the membrane due to conduction and the latent heat carried over by the trans-membrane vapor flow. Consequentially, the feed solution cools down from $T_{4}$ to $T_{6}$, whereas the permeate solution warms up from $T_{7}$ to $T_{9}$. To improve the energy efficiency of the MD operation, the effluent of the permeate solution is sent off to the HX where it exchanges the accumulated heat to the incoming stream of the feed solution $S_{1}$. The effluent temperature of the feed solution $T_{2}$ is still lower than the working temperature $T_{\mathrm{H}}$ even after acquiring the heat from HX hot stream. Therefore, $S_{2}$ absorbs a certain amount of heat $\left(q_{\mathrm{H}}\right)$ from the heat source to make up the temperature difference between $T_{2}$ and $T_{4}\left(=T_{H}\right)$. The energy efficiency of the thermal separation stage is quantified by the specific heat duty, $\beta$, which describes the amount of heat required to generate a unit mass of trans-membrane vapor:

$$
\beta \equiv \frac{q_{\mathrm{H}}}{\Delta Q_{\mathrm{MD}}}=\frac{q_{\mathrm{H}}}{Q_{11}}=\frac{c_{\mathrm{p}, 1}\left(T_{\mathrm{H}}-T_{2}\right)}{\gamma}
$$

where $c_{\mathrm{p}, 1}$ is the specific heat capacity of feed solution $S_{1}$. To maintain steady state operation and the maximum driving force in $\mathrm{MD}, S_{3}$ and $S_{6}$ are sent through the heat sinks to decrease their temperature to $T_{\mathrm{C}}$.

We also define an operating parameter, $\alpha$, which is the relative mass flow rate between the influents of permeate and feed solutions of the MD module:

$$
\alpha \equiv \frac{Q_{7}}{Q_{4}}=\frac{Q_{7}}{Q_{1}}
$$

This is an important parameter that will appear repeatedly in the following discussion. Hereafter, it is simply referred to as the relative flow rate.

Our recent modeling work on the MD-HX process reveals that both performance parameters $\gamma$ and $\beta$ are strongly dependent on $\alpha$, and that $\alpha$ can affect the operation regimes in the MD modules. ${ }^{34}$ With unlimited mass transfer kinetics in the MD module (i.e., sufficiently large MD membrane area, high membrane permeability), the MD operates in permeate limiting regime (PLR) when $\alpha$ is small or in feed limiting regime (FLR) when $\alpha$ is large. The PLR is defined such that the partial vapor pressure difference, which is the driving force for mass transfer, vanishes inside the module because the permeate temperature increases from $T_{\mathrm{C}}$ to $T_{\mathrm{H}}^{*}$, with $T_{\mathrm{H}}^{*}$ being the temperature of the pure permeate solution having the same partial vapor pressure $\left(p_{\mathrm{w}}\right)$ as the salty feed solution at $T_{\mathrm{H}}$ (i.e., $p_{\mathrm{w}}\left(0, T_{\mathrm{H}}^{*}\right)=p_{\mathrm{w}}\left(C_{\mathrm{F}}\right.$, $\left.T_{H}\right)$ ). Similarly, the FLR is defined such that the feed temperature decreases from $T_{\mathrm{H}}$, in the module, to $T_{\mathrm{C}}^{*}$, with $T_{\mathrm{C}}^{*}$ being the temperature of the salty feed solution that has the same partial vapor pressure as the pure permeate solution at $T_{\mathrm{C}}$ (i.e., $\left.p_{\mathrm{w}}\left(0, T_{\mathrm{C}}\right)=p_{\mathrm{w}}\left(C_{\mathrm{F}}, T_{\mathrm{C}}^{*}\right)\right)$. The PLR occurs when there is insufficient permeate flow compared to feed flow, as reflected by a small $\alpha$, whereas the FLR occurs when there is insufficient feed flow compared to permeate flow, as reflected by a large $\alpha$. In both cases the driving force falls to zero for a portion of the MD module. There exists a critical relative flow rate $\alpha^{*}$ at which the transition between PLR and FLR occurs. The critical relative flow rate $\alpha^{*}$ is a function of the working temperatures $T_{\mathrm{H}}$ and $T_{\mathrm{C}}$ and the working concentration $C_{1}$, factors that determine the thermodynamic properties of the solutions (e.g., specific heat capacity and latent heat).

When the mass transfer is limited by either insufficient membrane area or low membrane permeability, neither does the feed temperature reach $T_{\mathrm{C}}^{*}$, nor does the permeate temperature reach $T_{\mathrm{H}}^{*}$ in the MD module. Such an MD operation is defined to be in the mass transfer limited regime (MTLR). In-depth analysis of the operating regimes is presented in our recent publication. $^{34}$

Power Generation Stage. Streams $S_{10}$ and $S_{13}$ enter the PRO module as the high concentration draw solution and distilled water feed solution streams ("D" and "F" in Figure 1, respectively) in cocurrent mode with the draw solution chamber under a constant hydraulic pressure, $p_{\text {PRO }}$. The ideal semipermeable PRO membrane allows water permeation but rejects salts. The salinity difference between the feed and draw solution streams generates an osmotic driving force for water transport across the membrane, thereby increasing the draw solution flow rate by $\Delta Q_{\mathrm{PRO}}$, which is the integral of trans-membrane mass flux with respect to the entire membrane area of the PRO module. The PRO system generates power when a portion of the exit draw solution stream $\left(S_{16}\right)$, at the PRO working pressure $\left(p_{\text {PRO }}\right)$, is depressurized through the hydro-turbine to become $S_{19}$ at atmospheric pressure $\left(p_{0}\right)$. To maintain continuous operation, a pressure exchanger (PX) is employed to exchange 


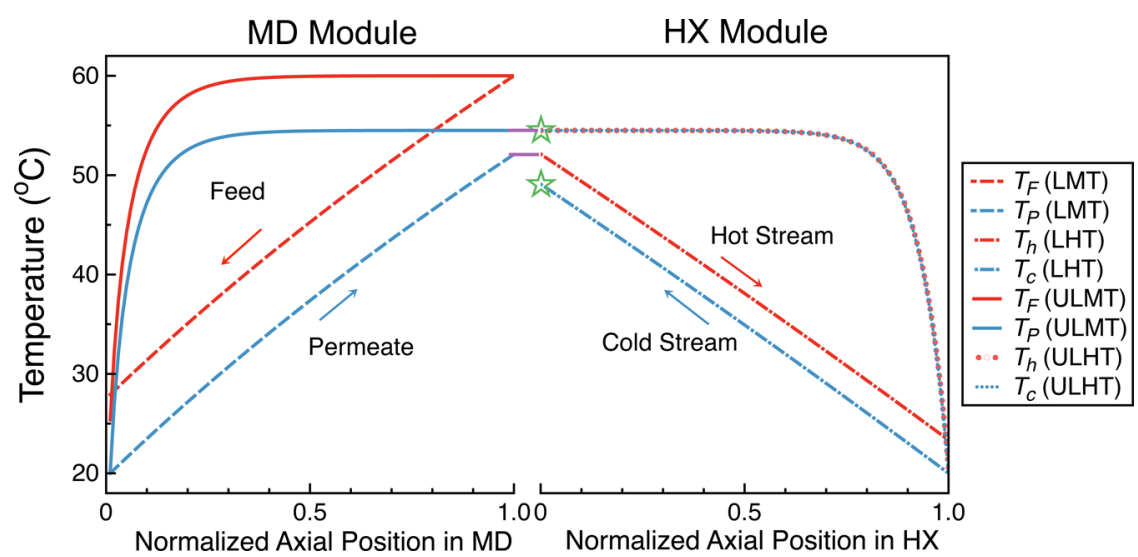

Figure 2. Temperature distribution profiles in the MD module (left) and HX module (right). The dashed lines in the MD module represent limited mass transfer (LMT), whereas the dash-dot lines in the HX module represent limited heat transfer (LHT). The solid lines in the MD module represent unlimited mass transfer (ULMT), while the dotted lines in the HX module (the two lines overlap) represent unlimited heat transfer (ULHT). $T_{\mathrm{F}}, T_{\mathrm{P}}, T_{\mathrm{h}, \text {, and }} T_{\mathrm{c}}$, are the local temperatures of the feed and permeate streams along the MD module and the local temperature of the hot and cold streams along the HX, respectively. The green stars indicate the temperature to which the exit HX cold stream reaches due to heat recovery. Note that the MD permeate effluent enters the $\mathrm{HX}$ as the hot stream influent and, therefore, is at the same temperature, as indicated by the purple connecting lines.

the heightened pressure of $S_{14}$ to the incoming draw solution stream $S_{8}$.

It can be proved (Supporting Information) that for the given configuration energy extraction is maximized in the power generation stage if all of the distillate from the thermal separation stage permeates through the PRO membrane (i.e., $\left.Q_{11}=\Delta Q_{\mathrm{PRO}}\right)$, even though a higher degree of permeation requires a lower applied hydraulic pressure in the draw solution chamber $\left(p_{\mathrm{PRO}}\right)$. Another condition for maximum energy output of the PRO system is that the applied pressure $p_{\text {PRO }}$ is equal to the osmotic pressure of the exiting draw solution $\pi\left(C_{\mathrm{D}}^{\mathrm{f}}\right)$, in which case the theoretical maximum extractable power $\left(P_{\max }\right)$ from the power generation stage is

$$
P_{\max }=p_{\mathrm{PRO}} \Delta Q_{\mathrm{PRO}}^{\mathrm{v}}=\pi\left(C_{\mathrm{D}}^{\mathrm{f}}\right) \Delta Q_{\mathrm{PRO}}^{\mathrm{v}}=\pi\left(C_{1}\right) \frac{\gamma Q_{1}}{\rho_{\mathrm{W}}}
$$

Here, $\pi(C)$ is the osmotic pressure of a solution of solute concentration $C$ (in molality), $C_{\mathrm{D}}^{\mathrm{f}}$ is the concentration of the diluted draw solution exiting the PRO module ( $\mathrm{f}$ for final), and $\Delta Q_{\mathrm{PRO}}^{\mathrm{v}}$ is the volumetric trans-membrane flow rate in PRO. Note that $C_{\mathrm{D}}^{\mathrm{f}}$ is also equal to the working concentration of the system, $C_{1}$. Because the closed-loop OHE system operates at steady state, mass balance rules that $\Delta Q_{\mathrm{PRO}}=\Delta Q_{\mathrm{MD}}=\gamma Q_{1}$. Equation 4 gives the maximum power attainable with constant pressure PRO, which is used for the following calculations to understand the highest efficiency of the hybrid system. However, we note that the operating condition to achieve maximum efficiency, $p_{\text {PRO }}=\pi\left(C_{1}\right)$, is inconsistent with the objective to attain peak membrane power density (power generation per unit membrane area). Hence, if power density is to be maximized for operational requirements, the extractable power will be lower than $P_{\max }$.

Energy Efficiency of the Overall System. The analyses of the thermal separation and energy generation stages in the previous section show that the hybrid system consumes thermal energy $\left(q_{\mathrm{H}}\right)$ to separate the distillate $\left(S_{11}\right)$ from the mixed solution $\left(S_{1}\right)$ and generates power $\left(P_{\max }\right)$ from mixing the distillate $\left(S_{11}\right)$ with the pressurized concentrated solution $\left(S_{10}\right)$. Therefore, the efficiency of the hybrid system as a heat engine, $\eta_{\mathrm{OHE}}$, can be calculated by combining eqs 2 and 4 :

$$
\eta_{\mathrm{OHE}}=\frac{P_{\max }}{q_{\mathrm{H}}}=\frac{1}{\rho_{\mathrm{w}}} \frac{\pi\left(C_{1}\right)}{\beta}=\frac{\gamma}{\rho_{\mathrm{w}} c_{\mathrm{p}, 1}} \frac{\pi\left(C_{1}\right)}{\left(T_{\mathrm{H}}-T_{2}\right)}
$$

which is dimensionless and independent of the scale of the system (i.e., the change in flow rate of $S_{1}$ has no impact on $\left.\eta_{\text {OHE }}\right)$.

Equation 5 predicts the efficiency of an ideal OHE of the design presented in Figure 1. For a given working concentration, the osmotic pressure $\pi\left(C_{1}\right)$ is constant, which implies that the maximum power extractable from the PRO per unit of distillate $\left(S_{11}\right)$ is constant (eq 4). In this case, it is the energy efficiency of the thermal separation, as reflected by the specific heat duty, $\beta$, that dictates the energy efficiency of the OHE. Our previous analysis on MD-HX indicated that, regardless of the mass transfer kinetics in MD and the heat transfer kinetics in $\mathrm{HX}$, the specific heat duty $\beta$ is a convex function of $\alpha$ that always reaches a minimum when $\alpha=\alpha^{*}$. ${ }^{34}$ Therefore, the hybrid system is anticipated to also achieve maximum energy efficiency at the critical relative flow rate $\alpha^{*}$.

\section{IMPORTANCE OF MASS AND HEAT TRANSFER KINETICS}

The mass transfer kinetics in MD and the heat transfer kinetics in HX have significant impacts on the specific heat duty, $\beta$, of the MD-HX system and, consequently, the energy efficiency of the PRO-MD hybrid system. To generalize the analysis, we quantify the mass transfer kinetics of MD by defining a mass transfer parameter $\chi:^{34}$

$$
\chi \equiv \frac{K_{\mathrm{m}, \mathrm{MD}} A_{\mathrm{MD}}}{Q_{\mathrm{F}}^{0}}
$$

where $K_{\mathrm{m}, \mathrm{MD}}$ is the mass transfer coefficient of the MD membrane, $A_{\mathrm{MD}}$ is the area of the MD module, and $Q_{\mathrm{F}}^{0}$ is the mass flow rate of the feed influent $\left(Q_{4}\right.$ in Figure 1$)$. A higher $\chi$ signifies faster overall mass transfer kinetics. Similarly, we can also quantify the heat transfer kinetics in the HX module by defining a heat transfer parameter $v:{ }^{34}$

$$
v \equiv \frac{K_{c, \mathrm{HX}} A_{\mathrm{HX}}}{\mathrm{Q}_{\mathrm{F}}^{0}}
$$



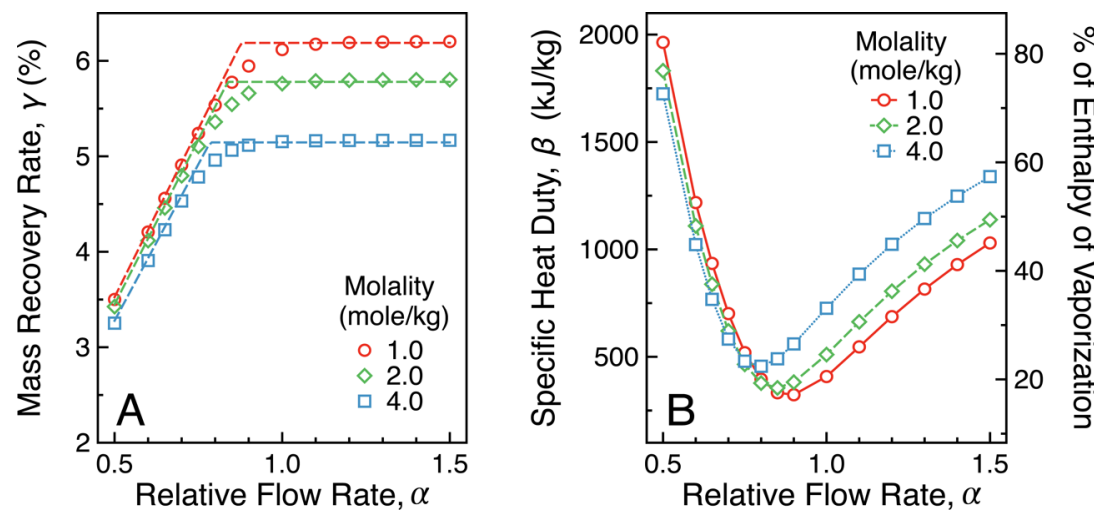

Figure 3. Simulation results of the MD-HX system with practical mass and heat transfer coefficients, for working solution concentrations of 1.0, 2.0, and $4.0 \mathrm{~mol} / \mathrm{kg} \mathrm{NaCl}$ (open symbols). (A) Mass recovery rate as a function of the relative flow rate, $\alpha$. The dashed lines are the maximum mass recovery rates obtainable as determined by thermodynamic analysis, which implicitly assume unlimited mass and heat transfer kinetics and are presented here for comparison. (B) Specific heat duty as a function of the relative flow rate, $\alpha$ (lines are drawn to guide the eyes). In these model simulations, the mass transfer parameter in the MD module $\chi=0.03{ }^{\circ} \mathrm{C}^{-1}$ and the heat transfer parameter $v=60 \mathrm{~kJ} \cdot \mathrm{kg}^{-1} \cdot{ }^{\circ} \mathrm{C}^{-1}$. The temperatures of the heat source and sink are 60 and $20^{\circ} \mathrm{C}$, respectively.

where $K_{\mathrm{c}, \mathrm{HX}}$ is the heat transfer coefficient of the $\mathrm{HX}$, and $A_{\mathrm{HX}}$ is the HX area.

Inadequate mass and heat transfers represent kinetic inefficiencies that lower the overall energy conversion performance and restrict the hybrid system from achieving the theoretical maximum efficiency. An inspection of eq 2 shows that a minimum specific heat duty $\beta$ can be achieved by using the least heat input $\left(q_{\mathrm{H}}\right)$ to attain the highest possible extent of separation $(\gamma)$. To understand this argument in detail, it is useful to compare the temperature distributions in the MD and $\mathrm{HX}$ modules between the case where both mass and heat transfer kinetics are limited and that where both are unlimited (Figure 2).

As described earlier, $\mathrm{HX}$ is employed to transfer the heat accumulated in the MD permeate effluent to bring $S_{1}$ from $T_{\mathrm{C}}$ to $T_{\mathrm{H}}$. Therefore, it is beneficial for the heat recovery to attain a high cold stream effluent temperature $\left(T_{2}\right.$ in Figure 1 , green stars in Figure 2), as the required heat input is proportional to $T_{\mathrm{H}}-T_{2}$ (eq 2). When heat transfer rate in the HX is limited due to a low $v$, the cold stream effluent temperature $\left(T_{2}\right)$ is lower than the hot stream influent temperature $\left(T_{9}\right)$. However, if $v$ is sufficiently large and heat transfer rate in $\mathrm{HX}$ is unlimited, $T_{2}$ can theoretically approach $T_{9}$ (green star on the same level as purple connecting line in Figure 2), thereby minimizing the heat required to make up the temperature difference between $T_{2}$ and $T_{\mathrm{H}}$.

Similarly, inadequate mass transfer rate in the MD module can detrimentally affect process performance. If $\chi$ is too small and, therefore, limits mass transfer in $\mathrm{MD}$, the operation cannot reach the PLR. As a result, the permeate effluent temperature $\left(T_{9}\right)$ does not reach $T_{\mathrm{H}}^{*}$, which is the highest temperature attainable by the permeate effluent when MD operates in PLR ${ }^{34}$ Therefore, both enhanced heat transfer kinetics in HX and mass transfer kinetics in $\mathrm{MD}$ raise the effluent temperature of the $\mathrm{HX}$ cold stream, $T_{2}$ (green stars in Figure 2). This, in turn, results in a lower $q_{\mathrm{H}}$ that eventually translates to a lower specific heat duty and a higher OHE energy efficiency.

Relatively faster MD mass transfer kinetics have another positive impact on the energy efficiency via increasing the mass recovery rate, $\gamma$. Our previous study demonstrated that for a given set of operating conditions (i.e., $T_{\mathrm{H}}, T_{\mathrm{C}}, C_{\mathrm{F}}$, and $\alpha$ ), there is a maximum mass recovery rate, $\gamma_{\max }$ which represents the thermodynamic limit attainable only with unlimited mass transfer kinetics. ${ }^{34}$ In other words, enhancing the MD mass transfer kinetics increases $\gamma$ to approach $\gamma_{\max }$. The improved mass recovery also reduces the specific heat duty, according to eq 2, and eventually leads to a higher energy conversion efficiency of the hybrid system. In the following sections, we first examine the performance of the OHE with limited mass and heat transfer kinetics. Next, the mass and heat transfer restrictions are removed to evaluate the thermodynamic limit of the system.

\section{PERFORMANCE WITH LIMITED MASS AND HEAT TRANSFER}

Energy Requirement of the Thermal Separation Stage (MD-HX). The relative flow rate $\alpha$ has an important impact on the performance of the MD-HX, which is illustrated by the change in the performance parameters $\gamma$ and $\beta$ as a function of $\alpha$ (Figure 3), as determined using the same numerical modeling conducted in our previous study on desalination by MD. ${ }^{34}$ The numerical results suggest that the mass recovery rate, $\gamma$, increases linearly with increasing $\alpha$ when $\alpha<\alpha^{*}$ (i.e., in PLR) and becomes independent of $\alpha$ when $\alpha>\alpha^{*}$ (i.e., in FLR) (Figure $3 \mathrm{~A}$ ). These numerical results (open symbols in Figure $3 \mathrm{~A}$ ) can be well fitted by analytical expressions derived on the basis of thermodynamic principles (dashed lines in Figure 3A) assuming unlimited mass and heat transfer kinetics (expressions presented in Supporting Information), except when $\alpha \approx \alpha^{*}$ (i.e., in MTLR). The numerical results deviate from the analytical expressions in MTLR because the analytical expressions were obtained from thermodynamic analysis that is applicable only when mass transfer proceeds to the extent that the thermodynamic driving force vanishes, which is not the case for MTLR that signifies an incomplete mass transfer. ${ }^{34}$ Throughout the range of relative flow rate, the mass recovery rate is always higher with lower $\mathrm{MD}$ feed concentration, $C_{1}$, which is primarily attributed to the dependence of heat capacity on $C_{1}$.

The specific heat duty $\beta$ (primary vertical axis on the left) and $\beta$ as a percent of the enthalpy of vaporization (secondary vertical axis on the right) as a function of $\alpha$ are shown in Figure 3B. The specific heat duty always reaches minimum when $\alpha=\alpha^{*}$, regardless of the working concentrations and temperatures. 
Detailed discussion on this dependence can be found in our previous MD-HX modeling paper. ${ }^{34}$ Briefly, when $\alpha<\alpha^{*}$, the heat recovery is limited by the mass flow rate of the hot stream, while when $\alpha>\alpha^{*}$, the heat recovery is limited by the highest temperature of the hot stream. Only when $\alpha=\alpha^{*}$, the heat recovery is optimal and the largest amount of heat can be recovered in the $\mathrm{HX}$, which leads to the most energy efficient operation. The critical relative flow rate, $\alpha^{*}$, is dependent on the feed concentration, $C_{1}$, because $C_{1}$, together with the working temperatures $T_{\mathrm{H}}$ and $T_{\mathrm{C}}$, determine the thermodynamic properties of solutions (e.g., heat capacity and latent heat) from which $\alpha^{*}$ is calculated. The analytical expression for $\alpha^{*}$ and its detailed derivation are presented in our recent publication on module scale modeling of the MD process. ${ }^{34}$

Overall Energy Efficiency of the Hybrid System. Once the specific heat duty, $\beta$, is obtained from the modeling of the thermal separation stage, the energy efficiency of the hybrid system, $\eta_{\mathrm{OHE}}$, can be readily calculated using eq 5 . Being inversely proportional to $\beta$ (eq 5), $\eta_{\mathrm{OHE}}$ is a concave function of the relative flow rate $\alpha$ with a maximum at $\alpha^{*}$ (Figure 4, primary

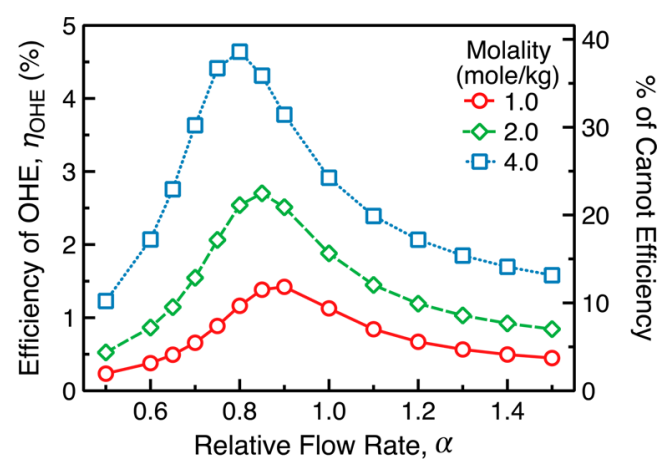

Figure 4. Energy efficiency of the PRO-MD hybrid system at different $\alpha$ and various concentrations of the working solution $(\mathrm{NaCl})$, as calculated using eq 5 and the specific heat duties presented in Figure $3 \mathrm{~B}$. The temperatures of the heat source and sink are 60 and $20^{\circ} \mathrm{C}$, respectively. The energy efficiency is presented as percent of the Carnot efficiency on the right axis. The Carnot efficiency for the working temperatures $\left(60\right.$ and $\left.20^{\circ} \mathrm{C}\right)$ is $12 \%$. The lines are for guiding the eyes.

vertical axis), which is dependent on $C_{1}$. However, although a higher MD feed concentration leads to a higher $\beta$ (Figure $3 \mathrm{~B}$ ), it also results in a higher $\eta_{\mathrm{OHE}}$, because the positive impact of a higher osmotic pressure $\pi\left(C_{1}\right)$ on $\eta_{\mathrm{OHE}}$ outweighs the negative impact of a higher specific heat duty that affects the energy requirement in the thermal separation stage (eq 5). The power generated from the PRO-MD hybrid system is plotted as a percentage of the Carnot efficiency, which is the highest energy conversion efficiency theoretically possible for a heat engine with given working temperatures based on the second law of thermodynamics $\left(\eta_{\text {Carnot }}=1-T_{\mathrm{C}} / T_{\mathrm{H}}\right)$. With the assumptions of finite MD membrane and $\mathrm{HX}$ areas, which are signified by moderate $\chi$ and $v$ values, the system (with $C_{1}=4 \mathrm{~kg} / \mathrm{mol}$ ) can achieve energy efficiency greater than $38 \%$ of $\eta_{\text {Carnot }}$.

\section{THERMODYNAMIC LIMIT OF PRO-MD POWER GENERATION EFFICIENCY}

The analysis presented in the previous section assumes limited mass transfer kinetics in $\mathrm{MD}$, which renders the MD operation in the MTLR when $\alpha$ is close to $\alpha^{*}$. We also assumed an HX with limited heat transfer kinetics so that the latent heat accumulated in the permeate stream, which enters the HX as the hot stream, cannot be completely recovered. Both these limitations represent process inefficiencies, and consequently, the specific heat duties shown in Figure $3 \mathrm{~B}$ and energy efficiencies in Figure 4 are below the thermodynamic limits when the system operates in MTLR. With unlimited mass and heat transfer kinetics assumed for the MD-HX system, the specific heat duty can approach the thermodynamic limit, which can be readily calculated from analytical expressions derived on the basis of thermodynamic principles.

Inspection of eq 2 shows that, for a given system (i.e., $T_{\mathrm{H}}, T_{\mathrm{C}}$, and $C_{1}$ ), increasing $T_{2}$ and $\gamma$ can both contribute to reducing $\beta$. The highest value that $T_{2}$ can reach is $T_{\mathrm{H}}^{*}$, which occurs only when MD operates in PLR. On the other hand, the upper limit of $\gamma$ is $\gamma_{\max }$ which occurs when MD operates in FLR (as illustrated in Figure 3A). Only when MD simultaneously operates in FLR and PLR can both the above conditions be met, which requires that (i) $\alpha=\alpha^{*}$ and (ii) mass/heat transfer kinetics are unlimited in MD-HX. From eq 2, the ultimate thermodynamic limit of minimum specific duty, $\beta_{\mathrm{min}}^{*}$, is thus

$$
\beta_{\min }^{*}=\frac{c_{\mathrm{p}, 1}\left(T_{\mathrm{H}}-T_{\mathrm{H}}^{*}\right)}{\gamma_{\max }}
$$
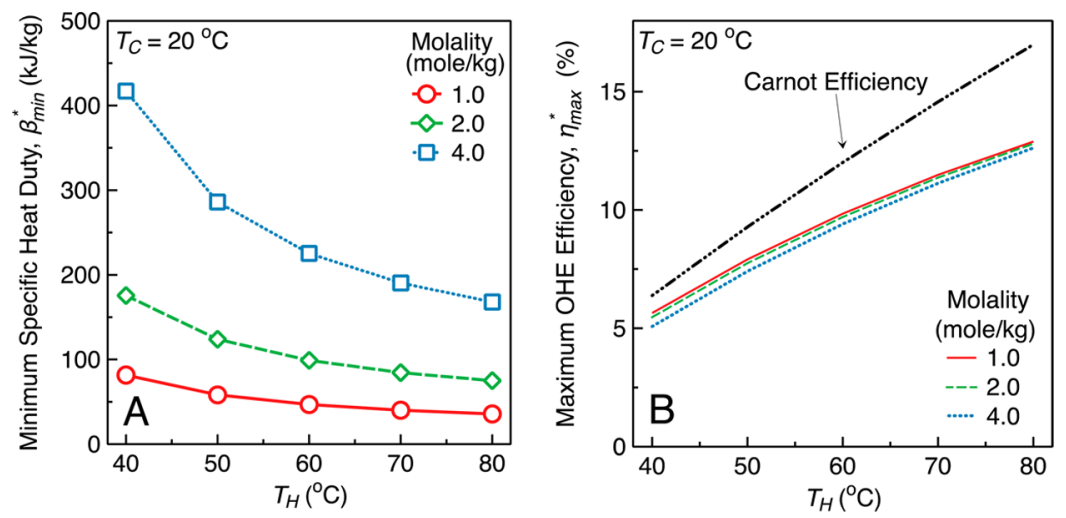

Figure 5. (A) Minimum specific heat duty, $\beta_{\min }^{*}$ of the thermal separation component (MD-HX). (B) Maximum energy efficiency, $\eta_{\max }^{*}$ of the hybrid system; the Carnot efficiency is also presented for reference. Mass and heat transfer kinetics in all modules of the system were assumed to be nonlimiting, and $\beta_{\min }^{*}$ and $\eta_{\max }^{*}$ are determined solely by the thermodynamic properties of the system. Both plots are presented as a function of the temperature of the heat source, $T_{\mathrm{H}}$, for feed stream $\mathrm{NaCl}$ concentrations of $1.0,2.0$, and $4.0 \mathrm{~mol} / \mathrm{kg}$. 
Note that the critical relative flow rate, $\alpha^{*}$, and all parameters on the right-hand side of eq 8 are functions of $T_{\mathrm{H}}, T_{\mathrm{C}}$, and $C_{1}$; therefore, $\beta_{\min }^{*}$ is also determined by $T_{\mathrm{H}}, T_{\mathrm{C}}$, and $C_{1}$. The dependences of $\alpha^{*}, T_{\mathrm{H}}-T_{\mathrm{H}}^{*}$, and $\gamma_{\max }$ on the temperature of the heat source, $T_{\mathrm{H}}$, and the working concentration, $C_{1}$, are presented in Figure S1 of the Supporting Information.

Our previous numerical modeling study on the MD-HX system demonstrated that the specific heat duty, $\beta$, always reaches a minimum when $\alpha=\alpha^{*}$, regardless of the mass and heat transfer kinetics. ${ }^{34}$ Therefore, the analytical evaluation of $\alpha^{*}$ allows facile determination of the optimal condition for the most energy efficient MD-HX operation. The minimum specific heat duty, $\beta_{\mathrm{min}}^{*}$, is plotted in Figure $5 \mathrm{~A}$ as a function of $T_{\mathrm{H}}$. Similar to the preceding discussions, the performance of the $\mathrm{MD}-\mathrm{HX}$ with the same working solution concentrations of 1.0, 2.0 , and $4.0 \mathrm{~mol} / \mathrm{kg}$ was examined. Figure $5 \mathrm{~A}$ shows that $\beta_{\min }^{*}$ is a decreasing function of $T_{\mathrm{H}}$ but an increasing function of $C_{1}$.

Substituting eq 8 into eq 5, we can analytically determine the maximum energy conversion efficiency, $\eta_{\max }^{*}$ of the hybrid system:

$$
\eta_{\max }^{*}=\frac{\gamma_{\max }}{\rho_{\mathrm{w}} c_{\mathrm{p}, 1}} \frac{\pi\left(C_{1}\right)}{\left(T_{\mathrm{H}}-T_{\mathrm{H}}^{*}\right)}
$$

Figure 5B shows $\eta_{\max }^{*}$ as a function of $T_{\mathrm{H}}$, with $T_{\mathrm{C}}$ fixed at $20^{\circ} \mathrm{C}$. The Carnot efficiency is also presented for comparison. The theoretical maximum efficiency of the hybrid system, $\eta_{\max }^{*}$ increases with increasing $T_{\mathrm{H}}$, which is common to all heat engines as larger temperature difference between the heat source and the heat sink always leads to a higher energy efficiency. It is observed that $\eta_{\max }^{*}$ is barely dependent on $C_{1}$ (Figure 5B), as compared to the strong dependence of $\eta_{\mathrm{OHE}}$ on $C_{1}$ (Figure 4). The difference between the concentration dependences of $\eta_{\max }^{*}$ and $\eta_{\mathrm{OHE}}$ is explained in detail in the Supporting Information.

In all cases, $\eta_{\max }^{*}$ is lower than the Carnot efficiency, $\eta_{\text {Carnot }}$ calculated using the working temperatures $T_{\mathrm{H}}$ and $T_{\mathrm{C}}$, as $\eta_{\text {Carnot }}$ can be achieved only when all processes involved are thermodynamically reversible. The difference between $\eta_{\max }^{*}$ and $\eta_{\text {Carnot }}$ is attributed to three sources of energy loss. The first source, which is dominant, is the residual enthalpy of the MD feed effluent and HX hot stream effluent $\left(S_{6}\right.$ and $S_{3}$, respectively, in Figure 1) that is not utilized in the configuration presented. Extra heat in excess of $T_{C}$ has to be released to the heat sinks for $S_{3}$ and $S_{6}$ to reach $T_{\mathrm{C}}$ and maintain steady state operation. The second source is the energy loss in PRO due to the constant pressure operation, which is an irreversible thermodynamic process that generates entropy. ${ }^{18}$ The last source of energy loss, which is a relatively small contribution, stems from the inevitable conductive heat transfer in MD, even if an extremely insulating membrane is assumed (as in this study). ${ }^{34}$ If a practical level of heat conduction is considered in the MD module, the energy efficiency of the hybrid system should be considerably lower.

The presented model yields the theoretical maximum energy conversion efficiency of a PRO-MD system (Figure 5B) that is relatively high compared to the Carnot efficiency, the theoretical upper bound of energy efficiency for any heat engine. In practice, however, there are several factors that prevent us from achieving such a theoretical limit. First, the maximum energy efficiency is attainable only with infinitesimal driving forces for mass transfer in MD and PRO and for heat transfer in HX. Practical operation, however, involves finite driving forces for efficient mass and heat transfers that are more economically justified, which inevitably reduces the energy efficiency of the system, as evident from comparing Figures 4 and 5B. Second, nonidealities relating to mass and heat transfer in each process, such as conductive heat loss in $\mathrm{MD}$, concentration polarizations and reverse salt flux in PRO, and imperfect $\mathrm{HX}$, compromise the overall energy efficiency to different extents. Last, energy losses due to mechanical nonidealities such as PX and TB inefficiency, as well as the energy spent for flow circulation, need to be accounted for when calculating the net energy output. Fully incorporating these practical factors, however, requires very detailed and system-specific modeling that is beyond the scope of the current study, which is to evaluate the thermodynamic limit of the energy efficiency.

\section{IMPLICATIONS FOR ENERGY PRODUCTION WITH LOW-GRADE HEAT}

Practical losses in an actual process and the intrinsic thermodynamic limitations of using a low-temperature heat source restrict energy production with low-grade heat to inherently low efficiencies, and only a fraction of the abundant quantity of low-temperature waste heat and geothermal energy can feasibly be converted to useful work. The intrinsically low efficiency implies that it would be more prudent to utilize lowtemperature thermal resources directly, for examples, in heat pumps and space heating. However, such direct uses are spatially confined to the locality of the low-grade heat source. In the absence of a matching need for direct use at the source, converting low-grade heat to electricity for conveyance is the only available option to exploit the thermal energy.

A prime factor to consider when selecting the technology to harness low-temperature heat sources for power generation is, therefore, the conversion efficiency. The thermodynamic and energy analysis performed in this study demonstrates that the hybrid PRO-MD power generation system is theoretically capable of achieving 9-10\% efficiency with low-grade heat at 60 ${ }^{\circ} \mathrm{C}$ (i.e., $75-83 \%$ of $\eta_{\text {Carnot }}$ ). Even with limited mass and heat transfer, the conversion efficiency is still comparable to that of the binary cycle power plants. More importantly, the PRO-MD system has the distinct advantage of being able to utilize lowgrade heat sources $<80{ }^{\circ} \mathrm{C}$, a region not easily accessible by organic Rankine cycle heat engines due to operational constraints imposed by the working fluids.

To advance OHE beyond conceptualization, more research is necessary to identify and close potential technology gaps. For instance, in the PRO membrane module, the draw side is pressurized to the osmotic pressure of the diluted working solution (i.e., $p_{\text {PRO }}$ ). For the 1,2 , and $4 \mathrm{~mol} / \mathrm{kg} \mathrm{NaCl}$ working solution examined in this study, the hydraulic pressures acting on the PRO membrane are approximately 46, 100, and 220 bar (667, 1450, and 3191 psi), respectively. Therefore, the development of mechanically robust PRO membrane modules capable of withstanding intense pressures is needed to take advantage of the energy efficiency benefits offered by a high working concentration. A recent study demonstrated a lab-scale operation of PRO with $3 \mathrm{M} \mathrm{NaCl}$ at an applied hydraulic pressure of $48 \mathrm{bar}^{35}$ which is promising for the realization of the PRO-MD system. Further studies are needed for a more thorough understanding of the energy efficiency with the system nonidealities accounted and for a rational economic analysis about the inherent trade-off between energy efficiency and power density, to advance power generation from low-grade heat using PRO-MD toward actual implementation. 


\section{ASSOCIATED CONTENT}

\section{S Supporting Information}

Flow rates and properties of different streams in the schematic diagram Figure 1 (Table S1). Governing equations for the mass and heat transfer in membrane distillation and heat exchanger modules (eqs S1-S12); derivation of the maximum power extractable in the hybrid system; analytical expression for the maximum mass recovery in direct contact membrane distillation; plot of the critical relative flow rate $\alpha^{*}$, minimum threshold temperature difference at the outlet of the $\mathrm{MD}$ permeate stream $\Delta T_{\min }=T_{\mathrm{H}}-T_{\mathrm{H}}^{*}$, and maximum mass recovery, $\gamma_{\max }$ as functions of heat source temperature $\Delta T_{\mathrm{H}}$ at different working concentrations (Figure S1); a detailed explanation on the difference between the dependences of $\eta_{\mathrm{OHE}}$ and $\eta_{\max }^{*}$ on $C_{1}$ (which includes Table S2, and Figures S2, S3, and S4). This material is available free of charge via the Internet at http://pubs.acs.org.

\section{AUTHOR INFORMATION}

\section{Corresponding Author}

*Tel: (203) 432-2789. E-mail: menachem.elimelech@yale.edu.

Notes

The authors declare no competing financial interest.

\section{ACKNOWLEDGMENTS}

We acknowledge the support received from the Advanced Research Projects Agency-Energy (ARPA-E), U.S. Department of Energy, via Grant DE-AR0000306. We also acknowledge a Graduate Fellowship (N.Y.Y.) made by the Environment and Water Industry Development Council of Singapore.

\section{REFERENCES}

(1) Chu, S.; Majumdar, A. Opportunities and challenges for a sustainable energy future. Nature 2012, 488 (7411), 294-303.

(2) Hendricks, T.; Choate, W. T. Engineering Scoping Study of Thermoelectric Generator Systems for Industrial Waste Heat Recovery; U.S. Department of Energy: Washington, DC, 2006.

(3) Key World Energy Statistics; International Energy Agency: Paris, 2012.

(4) Waste Heat to Power Systems; U.S. Environmental Protection Agency: Washington, DC, 2012; Combined Heat and Power Partnership.

(5) Tchanche, B. F.; Lambrinos, G.; Frangoudakis, A.; Papadakis, G. Low-grade heat conversion into power using organic Rankine cycles - A review of various applications. Renewable Sustainable Energy Rev. 2011, 15 (8), 3963-3979.

(6) Hettiarachchi, H. D. M.; Golubovic, M.; Worek, W. M.; Ikegami, Y. The performance of the kalina cycle system 11(KCS-11) with lowtemperature heat sources. J. Energy Resour. Technol. 2007, 129 (3), 243-247.

(7) Barbier, E. Geothermal energy technology and current status: an overview. Renewable Sustainable Energy Rev. 2002, 6 (1-2), 3-65.

(8) Chen, H. J.; Goswami, D. Y.; Stefanakos, E. K. A review of thermodynamic cycles and working fluids for the conversion of lowgrade heat. Renewable Sustainable Energy Rev. 2010, 14 (9), 3059-3067.

(9) Annual Energy Review; U.S. Energy Information Administration: Washington, DC, 2011.

(10) Holman, J. Perspective: Waste heat to power - still waiting for a breakthrough. Renewable Energy Strategies: Perspective 2011, No. EI229456.

(11) Dickson, M. H.; Fanelli, M. Geothermal Energy: Utilization and Technology; Earthscan: Sterling, VA, 2005; p xviii.

(12) Bertani, R. Geothermal power generation in the world 20052010 update report. Geothermics 2012, 41, 1-29.
(13) Geothermal Energy: The Potential for Clean Power from the Earth; Geothermal Energy Association: Washington, DC, 1999.

(14) Logan, B. E.; Elimelech, M. Membrane-based processes for sustainable power generation using water. Nature 2012, 488 (7411), 313-319.

(15) McGinnis, R. L.; McCutcheon, J. R.; Elimelech, M. A novel ammonia-carbon dioxide osmotic heat engine for power generation. $J$. Membr. Sci. 2007, 305 (1-2), 13-19.

(16) Ramon, G. Z.; Feinberg, B. J.; Hoek, E. M. V. Membrane-based production of salinity-gradient power. Energy Environ. Sci. 2011, 4 (11), $4423-4434$.

(17) Loeb, S. Osmotic power-plants. Science 1975, 189 (4203), 654655.

(18) Yip, N. Y.; Elimelech, M. Thermodynamic and energy efficiency analysis of power generation from natural salinity gradients by pressure retarded osmosis. Environ. Sci. Technol. 2012, 46 (9), 5230-5239.

(19) Achilli, A.; Childress, A. E. Pressure retarded osmosis: From the vision of Sidney Loeb to the first prototype installation - Review. Desalination 2010, 261 (3), 205-211.

(20) Lacey, R. E. Energy by reverse electrodialysis. Ocean Eng. 1980, 7 (1), $1-47$.

(21) Post, J. W.; Veerman, J.; Hamelers, H. V. M; Euverink, G. J. W.; Metz, S. J.; Nijmeijer, K.; Buisman, C. J. N. Salinity-gradient power: Evaluation of pressure-retarded osmosis and reverse electrodialysis. J. Membr. Sci. 2007, 288 (1-2), 218-230.

(22) Vermaas, D. A.; Saakes, M.; Nijmeijer, K. Doubled power density from salinity gradients at reduced intermembrane distance. Environ. Sci. Technol. 2011, 45 (16), 7089-7095.

(23) McGinnis, R. L.; Elimelech, M. Global challenges in energy and water supply: The promise of engineered osmosis. Environ. Sci. Technol. 2008, 42 (23), 8625-8629.

(24) Ikeda, M.; Miyamoto, K. Forward Osmosis Device, and Forward Osmosis Method. International Patent WO/2012/043669, 2012.

(25) Stone, M. L.; Rae, C.; Stewart, F. F.; Wilson, A. D. Switchable polarity solvents as draw solutes for forward osmosis. Desalination 2013, 312, 124-129.

(26) Wilson, A. D.; Stewart, F. F.; Stone, M. L. Methods and systems for treating liquids using switchable solvents. U.S. Patent 20130048561 A1, 2013

(27) Achilli, A.; Cath, T. Y.; Childress, A. E. Selection of inorganicbased draw solutions for forward osmosis applications. J. Membr. Sci. 2010, 364 (1-2), 233-241.

(28) Shaffer, D. L.; Yip, N. Y.; Gilron, J.; Elimelech, M. Seawater desalination for agriculture by integrated forward and reverse osmosis: Improved product water quality for potentially less energy. J. Membr. Sci. 2012, 415, 1-8.

(29) Curcio, E.; Drioli, E. Membrane distillation and related operations - A review. Sep. Purif. Rev. 2005, 34 (1), 35-86.

(30) Alkhudhiri, A.; Darwish, N.; Hilal, N. Membrane distillation: A comprehensive review. Desalination 2012, 287, 2-18.

(31) Khayet, M.; Matsuura, T. Membrane Distillation: Principles and Applications; Elsevier: Oxford, 2011.

(32) Veerman, J.; de Jong, R. M.; Saakes, M.; Metz, S. J.; Harmsen, G. J. Reverse electrodialysis: Comparison of six commercial membrane pairs on the thermodynamic efficiency and power density. J. Membr. Sci. 2009, 343 (1-2), 7-15.

(33) Długołęcki, P.s; Gambier, A.; Nijmeijer, K.; Wessling, M. Practical potential of reverse electrodialysis as process for sustainable energy generation. Environ. Sci. Technol. 2009, 43 (17), 6888-6894.

(34) Lin, S.; Yip, N. Y.; Elimelech, M. Direct contact membrane distillation with heat recovery-Thermodynamic insights from module scale modeling. J. Membr. Sci. 2014, 453, 498-515.

(35) Straub, A. P.; Yip, N. Y.; Elimelech, M. Raising the bar: Increased hydraulic pressure allows unprecedented high power densities in pressure-retarded osmosis. Environ. Sci. Technol. Lett. 2013, 1 (1), 5559. 